\title{
Research Progress of Cancer Stem Cells in Uveal Melanoma
}

\author{
Yu Ning Chen $(\mathbb{D}$ \\ Yang Li \\ Wen Bin Wei
}

Department of Ophthalmology, Beijing Tongren Eye Center, Beijing Key Laboratory of Intraocular Tumor Diagnosis and Treatment, Beijing Ophthalmology \& Visual Sciences Key Lab, Medical Artificial Intelligence Research and Verification Key Laboratory of the Ministry of Industry and Information Technology, Beijing Tongren Hospital, Capital Medical University,

Beijing, People's Republic of China
This article was published in the following Dove Press journal: OncoTargets and Therapy
Correspondence: Wen Bin Wei Department of Ophthalmology, Beijing Tongren Eye Center, Beijing Key Laboratory of Intraocular Tumor Diagnosis and Treatment, Beijing Ophthalmology \& Visual Sciences Key Lab, Medical Artificial Intelligence Research and Verification Key Laboratory of the Ministry of Industry and Information Technology, Beijing Tongren Hospital, Capital Medical University, I

Dong Jiao Min Lane, Beijing 100730

People's Republic of China

Tel +86-10-58269516

Fax +86-10-65 I25617

Email weiwenbintr@163.com
Abstract: Uveal melanoma is the most common malignant tumor in adult eyes, mostly in the choroid, but also in the iris and ciliary body. Distant metastasis is found in nearly half of the patients. Cancer stem cells are a kind of cells with the ability of self-renewal and multidirectional differentiation, which are related to tumor invasion and metastasis. Although the concept of cancer stem cells is relatively mature in other tumors, its existence and verification methods in uveal melanoma are still uncertain. A more in-depth understanding of cancer stem cells and their mechanism may reveal new strategies to treat uveal melanoma. This article reviews the concept of cancer stem cells and their research progress in uveal melanoma, including identification, probable markers, cancer stem cell targeted drug therapy and the controversies and prospects in this field.

Keywords: uveal melanoma, cancer stem cells, markers, drug treatment

\section{Introduction}

Although uveal melanoma (UM) is rarer than other tumors like lung cancer or skin melanoma, it is still the most common malignant tumor in adult eyes. The incidence of UM is associated with several individuals' backgrounds, including race, age, iris color, etc. ${ }^{1,2}$ For example, it is more prevalent in Caucasians with light irises than brown eyes. The annual incidence in European and American people is approximately 3.75-5.2 cases per million persons, ${ }^{3,4}$ which is significantly higher than that in Asians and Africans. About $50 \%$ of patients have metastases even if treated at the primary stage of the tumor, among which the liver is the most common site. Once metastasis, the median survival rate is 6 months. ${ }^{5}$ Although some eye-preserving treatments have emerged in recent years, such as radiotherapy, photodynamic therapy and transpupillary thermotherapy, ${ }^{6}$ the effects of these treatments are sometimes not ideal because of the limitations of understanding their pathogenesis. Cancer stem cell (CSC) is a cell concept that plays an important role in the occurrence and development of cancer at the end of the 20th century. This kind of cells have similar characteristics to normal stem cells and may serve as the origin of the tumor and promote its invasion and metastasis. Many studies have found that CSC is more resistant to treatment. Therefore, cancer stem cells have been a hot topic of research and therapeutic targets in last two decades. An increasing number of research is oriented toward UM-CSC in recent years, and this article reviews the research progress of the concept, markers and drug treatments of UM-CSC. 


\section{The Concept of Cancer Stem Cell}

In solid tumors, clinical manifestations such as recurrence, metastasis and drug resistance of resected tumors are often found, it is speculated that these may have a relation to a subset of tumor cells which are named as CSC. The concept of CSC was first proposed by Lapidot in 1994. Through the expression of specific markers, they screened a class of leukemic cells that have the ability of self-renewal and maintain malignant phenotype, and named them as acute myeloid leukemia stem cells, which confirmed the existence of CSC. In 2006, The American Association for Cancer Research defined CSC as a kind of cell within cancer that has the ability of self-renewal and is the cause of tumor heterogeneity, which is characterized by self-renewal and multidirectional differentiation. ${ }^{7} \mathrm{CSC}$ can differentiate into different subtypes of cancer cells. In addition, it also expresses lymphatic vessels and similar blood vessels' markers, which may be related to vasculogenic mimicry. ${ }^{8}$

Regarding the role of CSC in tumorigenesis and development, it is generally believed that all kinds of cancer cells come from a small number of CSC subsets, that is, CSC is the origin of cancer cells and is connected with tumor progression and curative effect, thus many studies have focused on targeted treatment of CSC to improve the prognosis of cancer therapy. At present, it is believed that there are two main mechanisms for the formation of CSC: one is from normal stem cells or their early progenitor cells, which stop differentiation and experience mutation to have an abnormal differentiation to form CSC at a specific stage of differentiation. The other mechanism is dedifferentiation from differentiated cells to CSC. ${ }^{9}$ In addition, CSC also has differentiation heterogeneity and plasticity, which is inseparable from the tumor microenvironment, gene mutations, and epigenetic modification ${ }^{10}$ (Figure 1). At present, most views tend to lean to the former, considering that abnormal differentiation may occur at any stage of normal proliferation and differentiation of stem cells. The cancer may show poor differentiation if this abnormal stem cells differentiation occurs in the early stage. While if it occurs in the late stage of stem cells differentiation, the tumor may be a highly differentiated cancer or benign tumor.

\section{Identification and Research Hotspots of Cancer Stem Cell}

Because CSC has the characteristics of multidirectional differentiation, self-renewal, tumorigenicity and drug resistance, lots of experiments tend to prove the above characteristics of CSC when proving the existence of CSC. There are currently no consensus CSC markers for melanoma. CD44+ cells seem to have stronger metastatic ability than CD44- cells in cutaneous melanoma (CM) cell line, and some people use CD133 and ALDH as markers to screen CM-CSC. The effect of drugs and its action pathway on UM-CSC were investigated by Pan and colleagues in China in which they used aldehyde dehydrogenase assay, transwell migration and invasion assay, melanosphere culture and xenograft experiment to identify the existence of $\mathrm{CSC}^{11,12}$ In addition, tumor
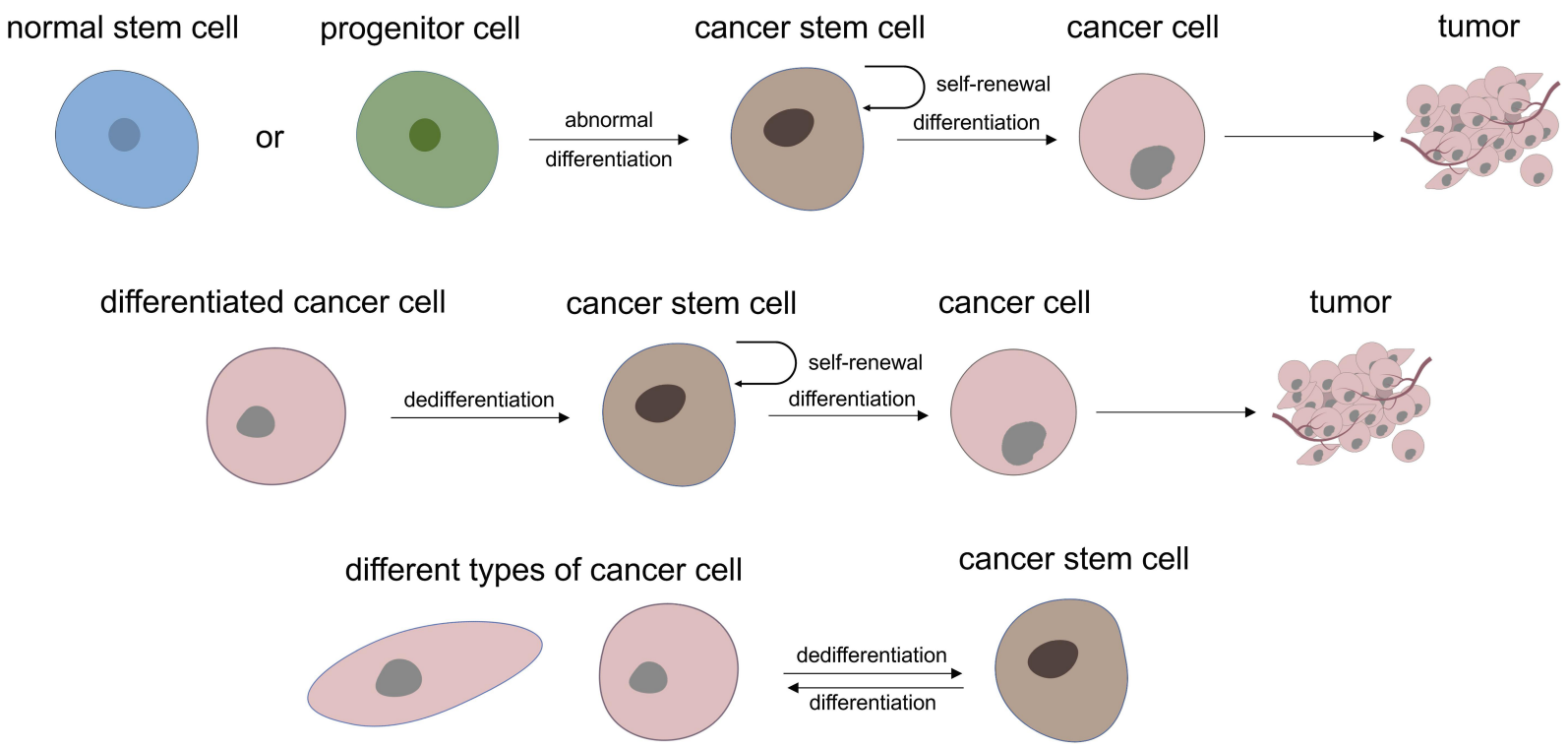

Figure I Two main theory of where cancer stem cells may be derived from and the heterogeneity and plasticity characteristic of UM-CSC. 
transendothelial migration assay, anoikis resistance assessment and other possible markers of UM-CSC (such as CD166, Nestin, etc.) were also used to identify CSC and its characteristics. ${ }^{13}$

There are three distinct clone types in tumor cell clonal culture: holoclones, meroclones and paraclones. Holoclones have a strong proliferation and tumorforming ability, paraclones' proliferation ability is the lowest, so it is believed that holoclones may be a kind of clone formed by CSC. Many subsequent CSC studies are based on this theory. Kalirai et $\mathrm{al}^{14}$ performed single-cell cloning adherence culture and nonadherent sphere culture experiments on UM cell lines (Mel270, Omm2.5) for primary and liver metastases, and demonstrated the existence of CSC in UM. It was found that all of the two cell lines showed three colony types during adherent culture. Continued passage and planting at low density of holoclone-derived and paraclones-derived cultures found that holoclones cells can differentiate into all three colony type, and the proportion of each type is similar to parental cells, while cells isolated from paraclones can only produce paraclones. Nonadherent sphere assay found that as the number of passages increased, the formation efficiency of melanocyte spheres increased. The third-generation melanocyte spheres cells of Mel270 and Omm2.5 showed morphologic characteristics consistent with all three colony types. Holoclones and MS cells were found antigenic heterogeneity, primitive migratory neural crest phenotype (PAX3, SOX10, SNAI2) and more differentiation phenotypes in subsequent experiments. The number of holoclones cells in two cell lines treated with cisplatin was significantly increased compared with untreated cells, which proved that $\mathrm{CSC}$ has the ability to resist chemotherapy. In the GEP classification of UM, Class 2 UM has a significantly worse prognosis than Class 1 , Chang et $\mathrm{al}^{15}$ tried to explain this phenomenon from the CSC perspective. They speculated that Class 2 tumor may contain primitive neuro/ectodermal stem cell-like cells which are responsible for tumor formation and metastasis. Therefore, gene set enrichment analysis found that the gene expression patterns of Class 2 UM cells and primitive ectoderm and neural crest stem/progenitor cells are statistically associated, while Class 1 UM cells only showed concordance with more mature cell types, such as committed neural crest cells and differentiated melanocytes. ${ }^{16}$ Inactivation of BAP1 is one of the characteristics of Class $2 \mathrm{UM}$, several studies indicated that BAP1 regulates the development and differentiation of uveal melanocyte lineage, BAP1 loss was related to the stem-like phenotype of UM cells. ${ }^{17,18}$

Besides the separation and identification of CSC, deeper research has also been gradually carried out in recent years. In view of the characteristics of CSC's self-renewal and multidirectional differentiation, the research of CSC targeted therapy is also increasing. ${ }^{12}$ It has also been suggested that cytokines, drug and radiation therapy in the tumor microenvironment will affect the cell phenotype and differentiation direction of CSC. ${ }^{19,20}$ Research on cell cycle, CSC plasticity and its pathways are also rapidly developing. Signal pathways such as Wnt, AKT, MERK and NF- $\kappa \mathrm{B}$ are hotspots in CSC research. ${ }^{21}$ With the development of single-cell sequencing technology, it may be easier to explain the heterogeneity of cancer by studying the characteristics of CSC at the single-cell level. ${ }^{22}$

\section{Possible Markers of UM Cancer Stem Cell}

At present, there is no recognized marker of melanoma stem cells, and the research on their pathway is even less. Although the CSC markers for glioma, colorectal cancer and other tumors are relatively clear, its mechanism is still uncertain. This review summarizes the CSC markers proposed and studied in previous UM-CSC articles (Table 1).

CD133, also known as PROM1, encodes prominin-1 protein, which is a transmembrane glycoprotein that is usually expressed in adult stem cells and maintains stem cell characteristics by suppressing differentiation. CD133 is the most widely used stem cell marker, which is expressed in both normal and cancer stem cells. CD133+ cells show stem cell-like characteristics in brain tumors, renal cancer, lung cancer and other types of tumors. ${ }^{29}$ CD133 not only activates signal molecules through direct interaction but also exerts the function of cancer stem cells by regulating cancer metabolism, ${ }^{30}$ it is even found that CD133 may be related to drug resistance-related gene ABCG2. CD133 can induce $\mathrm{Wnt} / \beta$-catenin signal transduction and play a regulatory role in $\mathrm{PI} 3 \mathrm{~K} / \mathrm{Akt}$ signaling pathway. Flow cytometry sorting showed that there were CD133+ and Nestin+ cell groups in UM cell lines Mel270, OMM 2.3 and OMM2.5. Immunohistochemistry showed that CD133+ UM cells were mainly localized at the invading tumor front. $^{23}$ It is speculated that UM liver metastasis may be connected with CSC because CD133+ CSC-like phenotype cancer cells disappeared with the progression of the tumor in UM liver metastasis. ${ }^{24}$ However, a meta-analysis of 299 
Table I CSC Markers Studied in UM

\begin{tabular}{|c|c|c|c|}
\hline Markers & Samples & Characteristics of Marker Positive Cells & References \\
\hline CDI33 & $\begin{array}{l}\text { Tumor tissue and cell lines (92.I, SP6.5/8.0, TP3I, } \\
\text { Mel270, OMMI/2.3/2.5) }\end{array}$ & $\begin{array}{l}\text { Mainly located at the leading edge of tumor invasion } \\
\text { The number of cells decreases with the development of } \\
\text { metastatic tumors }\end{array}$ & {$[23,24]$} \\
\hline CDI66 & $\begin{array}{l}\text { Tumor tissue and cell lines ( } 92.1, \text { Mel270, MP4I/46, } \\
\text { OMMI/2.3/2.5, MM66) }\end{array}$ & $\begin{array}{l}\text { The expression of CDI } 66 \text { is up-regulated in highly malignant } \\
\text { tumors and the process of anoikis resistance. }\end{array}$ & {$[13]$} \\
\hline Nestin & $\begin{array}{l}\text { Tumor tissue and cell lines (92.I, Mel270, MP4I/46, } \\
\text { OMMI/2.3/2.5, MM66, SP6.5/8.0, TP3I) }\end{array}$ & $\begin{array}{l}\text { Mainly located at the invading tumor front } \\
\text { Related to poor prognostic factors }\end{array}$ & {$[13,23,25]$} \\
\hline CD27I & Cell lines (C9I8, OCMI) & Involved in vasculogenesis & [26] \\
\hline ALDH & Cell lines (OMMI) & Have strong ability to form xenografted tumor & {$[11]$} \\
\hline SOX2 & $\begin{array}{l}\text { Tumor tissue and cell lines (92.I, SP6.5/8.0, TP3I, } \\
\text { Mel270, OMMI/2.3/2.5) }\end{array}$ & Mainly located at the invading tumor front & [23] \\
\hline ABCB5 & $\begin{array}{l}\text { Tumor tissue and cell lines (92.I, SP6.5/8.0, TP3I, } \\
\text { Mel270, OMMI/2.3/2.5) }\end{array}$ & Mainly located at the invading tumor front & [23] \\
\hline$A B C G 2$ & Tumor tissue & Expressed in most UM cells & [27] \\
\hline $\mathrm{EZH} 2$ & $\begin{array}{l}\text { Tumor tissue and cell lines (92.1, Mel270, MP4I, } \\
\text { OMMI/2.3) }\end{array}$ & $\begin{array}{l}\text { Promote cell growth, migration, and invasion, improved self- } \\
\text { renewal ability of CSC and its percentage }\end{array}$ & [II] \\
\hline JARIDIB & Tumor tissue & $\begin{array}{l}\text { High expression level is associated with more aggressive } \\
\text { phenotype }\end{array}$ & [28] \\
\hline OCT4 & Tumor tissue & Expressed in most UM cells & [27] \\
\hline CD44 & $\begin{array}{l}\text { Tumor tissue and cell lines (92.I, SP6.5/8.0, TP3I, } \\
\text { Mel270, OMMI/2.3/2.5) }\end{array}$ & Expressed in most UM cells & [23] \\
\hline
\end{tabular}

cases of cutaneous and uveal melanoma from five studies showed that $61.7 \%$ of the melanoma cells were CD133+, the author thought CD133 could not be used as a marker to recognize melanoma $\mathrm{CSC}$ at present. ${ }^{31}$

CD166, also known as ALCAM, is a member of the immunoglobulin superfamily and transmembrane protein. It is related to different stages of biological development, including neural development, branched organ development, hematopoiesis and immune response, and regulates the growth of retinal ganglion cell axons. CD166 is expressed on the surface of mesenchymal stem cells and human melanoma cell lines. A study on the expression of CD166 in CM cells has described its role in controlling the transition from local cell proliferation to tissue infiltration. $^{32}$ Djirackor et $\mathrm{al}^{13}$ found that the expression of CD166 in UM cells was 4 times higher than that in normal choroidal melanocytes. In the evaluation of apoptosis, UM cells up-regulated the average expression of CD166 during the period of resistance to apoptosis compared with adherent cells. Then they analyzed the $80 \mathrm{UM}$ cases information in TCGA database and found that the low expression of BAP1 gene and monosomy 3 had a relation to the high expression of CD166, indicating that the increased expression of CD166 gene was significantly related to tumor metastasis and the decrease of overall survival time. Therefore, CD166 was considered to be a suitable marker for UM-CSC.

Nestin is a member of the intermediate filament protein family, which was first found in the cytoplasm of neuroepithelial stem cells. It is also expressed in migration and proliferation cells during embryonic development. Nestin promotes the decomposition of phosphorylated vimentin intermediate filaments during mitosis and may participate in the transport and distribution of intermediate filaments and other cytokines to progeny cells during precursor cell division. It is also expressed in glioblastoma multiforme and UM, which seems to be related to the high proliferation and migration activity of these tumors. The expression of Nestin in metastatic UM cells was higher than that in primary UM and was connected to poor prognosis. ${ }^{13}$ In primary UM cells, Nestin was expressed in the cell membrane and cytoplasm, while in metastatic UM, it was mainly expressed in the cytoplasm. The high level of Nestin expression is associated with the short survival time and with known adverse prognostic factors in primary UM such as epithelioid cell morphology, high mitotic count, monosomy 3 and chromosome $8 \mathrm{q}$ gain. This study indicated the expression of CSC marker Nestin can predict tumor phenotype and prognosis. ${ }^{25}$ Flow cytometry showed that in three UM cell lines (Mel270, OMM2.3, OMM2.5), 
a small number of CD133+ cells also expressed Nestin, and no CD133+/Nestin- cells were found, speculating that the co-expression of CD133 and Nestin can be used as a marker for CSC screening. ${ }^{23}$

CD271, also known as NGFR, is a low-affinity nerve growth factor receptor, which has been found in several human nerve crest-derived tissues and some tumors, including melanoma. A study of CM published in Nature magazine showed that $\mathrm{CD} 271+$ cells had a higher incidence of CM than CD271- cells in immunodeficient mice, and tumors transplanted with CD271+ cells could metastasize in mice. Their team also found that the expression of other tumor antigens decreased or lost in most CD271+ cells, and speculated the melanoma cells of CD271+ may be in a state of incomplete differentiation. ${ }^{33}$ Because melanoma cells have similar characteristics to neural crest stem cells, CD271 may also be one of the markers of melanoma stem cells. Vasculogenic mimicry was first discovered in UM, and many subsequent researches on it are based on 3D cultures of tumor cells. Some studies have shown that CD271 is expressed in 3D cultured UM cell line C918 with vasculogenic mimicry, while monolayer adherent cultured UM cells do not express CD271. ${ }^{26}$ Recent studies have found NEK2, TOP2A and RAD51AP1 genes that respond to CD271 expression in metastatic $\mathrm{CM}$, these genes may be the potential drivers of CM metastasis. In addition, CD271 may be regulated by p53, while drugs related to DNA damage require p53dependent response, so it is considered that CD271 is associated with DNA repair, drug response and tumor metastasis. ${ }^{9}$

ALDH belongs to one of the proteins encoded by the aldehyde dehydrogenase family and participates in alcohol metabolism. It exists widely in normal tissue stem cells and can be used as a marker of cancer stem cells. In immunodeficient mice, $\mathrm{CM}$ cells of $\mathrm{ALDH}+$ are more tumorigenic and more resistant to chemotherapeutic agents than ALDH- cells. Silencing ALDH1A by siRNA or shRNA can lead to cell cycle arrest and apoptosis, decrease cell viability in vitro and tumorigenesis in vivo, and make $\mathrm{CM}$ cells more sensitive to drugs. ${ }^{34}$ In UM cell line OMM1, ALDH+ cells can form more subcutaneous xenografted tumors in NOD/SCID mice than ALDH- cells, which proves that ALDH+ cell subsets can enrich more CSC in UM. ${ }^{11}$ Several studies on targeted therapy of CM with ALDH isozyme have made good progress, which provides evidence for the treatment of CSC in the future. ${ }^{35,36}$
SOX2, a member of the transcription factor family of embryonic stem cells, is expressed in the nucleus and plays a key role in the development of embryonic stem cells and in maintaining the pluripotency and self-renewal of embryonic stem cells and some cell lines (such as nerve cells, osteoblasts and melanocytes derived from neural crest). In the study of CM-CSC by Santini et al, ${ }^{37}$ it was found that SOX2 was highly expressed in CSC, while the knockdown of SOX2 greatly reduced the self-renewal ability of CSC with high expression of ALDH, inhibited the growth of primary CM cells and induced apoptosis, which was confirmed in xenografted mice. In addition, they also found that SOX2 expression is regulated by $\mathrm{HH}-$ GLI signaling pathway in melanoma cells, and that transcription factors GLI1 and GLI2 downstream of HH-GLI signal bind to the proximal promoter region of SOX2 to regulate CM cell proliferation and CSC self-renewal. SOX2+ UM cells were also found to be mainly located in the front of tumor invasion. ${ }^{23}$ However, subsequent experiments showed SOX2 deletion does not affect the occurrence of $\mathrm{CM}$ nor its growth and metastasis. ${ }^{38,39}$ Therefore, whether SOX2 can be used as a CSC marker of melanoma still needs further study.

ABCB5, a member of the ATP-binding cassette transporter superfamily, is a transmembrane glycoprotein that mediates the transport of specific substances through the cell membrane, including some chemotherapeutic drugs. It is considered to be one of the CSC markers. One thing is that it has been found that $\mathrm{ABCB} 5+\mathrm{CM}-\mathrm{CSC}$ has higher metastatic potential than ABCB5- cells, and knocking down the expression of $\mathrm{ABCB} 5$ can not only reduce lung metastasis of $\mathrm{CM}$ xenografted mice but also migration and invasion of CM cells in vitro. There is a positive correlation between $\mathrm{ABCB} 5$ expression and NF- $\kappa \mathrm{B}$ p65 in $\mathrm{CM}$ and $\mathrm{CM}$ cell lines, so it is speculated that $\mathrm{ABCB} 5$ enhances the stability of $\mathrm{p} 65$ protein by inhibiting $\mathrm{p} 65$ ubiquitination, thus activating NF- $\kappa$ B pathway and promoting melanoma metastasis. ${ }^{40}$ As a direct target of microphthalmos-related transcription factor MITF, the expression of $\mathrm{ABCB} 5$ can be induced by the key activator and cofactor $\beta$-catenin of MITF. However, some studies have shown that ABCB5 is unlikely to be used as a CMCSC marker of dedifferentiation and may be related to the non-genetic resistance of highly differentiated $\mathrm{CM}^{41}$ In the UM-CSC study, ABCB5+ cells were found in rare pigmented and nonpigmented tumor cells, apart from they tended to be located at the front of tumor invasion. ${ }^{23}$ 
ABCG2 also belongs to the family of ATP-binding cassette transporters. As a membrane transporter, ABCG2 can pump chemotherapeutic drugs from intracellular to extracellular through the formation of functional dimers, resulting in chemotherapy resistance, which is considered to play an important role in multiple drug resistance of malignant tumors, so it is also called a multi-drug resistance transporter. Some studies have found that ABCG2 only expressed in early stem cells, with the differentiation of cells, ABCG2 expression gradually stops, so it is considered to be an important marker of stem cells. ABCG2 staining was positive in mixed primary $U M$ and expressed in cell membrane and cytoplasm, and there was functional ABCG2 transporter in UM cells. ${ }^{27}$ ABCG2 accumulates in the cytoplasm of poorly differentiated tumor cells with defects in PI3K signaling pathway. Co-expression of CD133 and ABCG2 was found in CM cell line WM115. These cells can form melanin spheres after suspension culture, express precursors and mature oligodendrocyte markers, and transdifferentiate into astrocytes or mesenchymal cells under specific growth conditions, indicating the multi-directional differentiation and self-renewal ability of these cells. ${ }^{42}$

EZH2 is the main catalytic protein of polycomb repressive complex 2 , which plays an important role in maintaining the characteristics of CSC. It is highly enriched in CSC of esophageal, gastric and prostate cancer, and plays a regulatory role in cytoskeleton related genes. ${ }^{43}$ EZH2 was overexpressed in UM and promoted the proliferation of it. In EZH2 exhausted UM cell lines, the percentage of $\mathrm{ALDH}+$ cells decreased, CSC self-renewal and continuous replication significantly decreased. The proportion of CSC was increased through miR-29c-DVL2- $\beta$-catenin signal pathway, and migration and invasion were promoted through RhoGDI $\gamma$-rac1 axis. ${ }^{11}$ A similar conclusion has been reached in CM-CSC study. ${ }^{44}$

JARID1B is a lysine-specific demethylase, which belongs to the JmjC domain protein family. JARID1B removes methyl residues from histone, which is convenient for RNA polymerase to recognize and bind DNA. Previous studies have found that JARID1B can be used as a CM cell marker of slow cell cycle. These cells have formed a subgroup of JARID1B + cells both in vivo and in vitro. This subgroup of cells has a strong ability of selfrenewal in vitro. Knocking down JARID1B in vitro will lead to CM cell exhaustion, and it has been found that JARID1B is necessary for the continuous growth and metastasis of xenografted melanoma. ${ }^{45}$ A study on the expression of JARID1B in UM found that although
JARID1B was expressed in most UM cells, the expression of JARID1B was higher in highly invasive UM, and progenitor cells were found in ciliary epithelium. ${ }^{28}$

OCT4 is a transcription factor containing POU homologous domain, which plays a key role in embryonic development and stem cell pluripotency. In 2006, scientists introduced four transcription factors, including OCT4, into differentiated somatic cells and obtained induced pluripotent stem cells. Kumar et $\mathrm{al}^{46}$ found that the expression of OCT4 gene or transmembrane transfection of OCT4 protein promoted the dedifferentiation of melanoma cells. After dedifferentiation, the expression of melanocyte markers decreased significantly and had sphere-forming ability, and the resistance to chemotherapy and hypoxia injury was significantly enhanced. These cells also acquired characteristics similar to those of CSC, such as the ability of multidirectional differentiation and the expression of $\mathrm{ABCB} 5$ and CD271, suggesting that CSC may be obtained through dedifferentiation. In a study of CM brain metastatic tumor tissues, it was found that OCT4 and other CSC markers were expressed in all metastatic tissues. ${ }^{47}$ In another study, down-regulation of genes related to pigmentation in UM was observed after comparing UM cells with normal melanocytes, and stem cell markers ABCG2 and OCT4 were expressed in most UM cells, suggesting that there were undifferentiated cells in $\mathrm{UM}^{27}$

Transmembrane glycoprotein CD44 can be used as a stem cell marker for a variety of normal and tumor tissues, and also interact with extracellular matrix components (including hyaluronic acid, collagen, fibronectin, integrin and laminin). These interactions induce changes in cytoskeleton and activation of signal pathways involved in cell adhesion and migration, which play a recognition role in the process of lymphocyte homing. It can be used as a downstream molecule of TGF- $\beta$ to participate in the EMT process. CD44 was highly expressed in most UM cell lines ${ }^{23}$ and primary UM cells, ${ }^{48}$ and the percentage of CD44+ cells was relatively high. Although CD44 can be used as CSC-marker in prostate cancer, gastric cancer, pancreatic cancer, ovarian cancer, etc., whether CD44 can be used as UM-CSC remains to be considered.

\section{Drug-Related Research of UM Cancer Stem Cell}

Because of the "seed" characteristic of CSC, more and more treatment studies focus on CSC. In recent years, it has been found that salinomycin can be used as a selective 
inhibitor of CSC in many cancer cell lines. In melanosphere formation assays of UM cell lines Mel270 and 92.1 treated with salinomycin, the size, number and replantation ability of melanosphere decreased significantly, and the percentage of ALDH+ cells were also decreased. It was found that salinomycin could eliminate UM-CSC in the mouse model of UM liver metastasis and effectively inhibit liver metastasis, they believed that Twistl was the basis of salinomycin-induced clearance of CSCs and inhibition of UM cell migration and invasion. ${ }^{12}$ In another study published by this team, NEDD8-activating enzyme inhibitor MLN4924 has been shown to inhibit UM-CSC properties by reducing Slug protein level. The results showed that MLN4924 inhibited the self-renewal ability of UM cells and decreased the percentage of ALDH+ UM cells. On the other hand, the decrease of melanosphere formation ability mediated by MLN4924 was improved by UM cells which forced to express slug. ${ }^{49}$ Similar methods also demonstrated that histone deacetylases inhibitor JSL-1 and antihelminthic drug niclosamide inhibit the selfrenewal ability of UM-CSC through Wnt/ $\beta$-catenin pathway, ${ }^{50,51}$ Cyclin-dependent kinase inhibitor SNS-032 inhibits the stem cell characteristics of UM-CSC by inhibiting the transcription of Kruppel-like transcription factor 4. ${ }^{52}$ Previous studies have shown that photodynamic therapy for UM in the short term is worth looking forward to. ${ }^{53}$ As a photosensitizer, verteporfin is effective in the treatment of age-related macular degeneration and choroidal neovascularization. After in-depth study, it was found that verteporfin can not only inhibit the migration and invasion of UM cells but also affect the stem cell characteristics of UM-CSC. ${ }^{54}$

In addition to drug-targeted treatment of $\mathrm{CSC}$, we can also consider blocking the signal transduction pathway, ie, $\mathrm{Wnt} / \beta$-catenin pathway, changing the tumor microenvironment, reducing the activity of CSC membrane transporters such as ATP-binding cassette transporter and inducing CSC differentiation.

\section{Controversy About UM Cancer Stem Cell}

Although the existence of CSC has been confirmed in multiple types of malignant tumors, the existence of CSC in cutaneous and uveal melanoma is still controversial. Some studies have shown that CM cells may change their phenotype according to the changes of the surrounding microenvironment because melanocytes originate from nerve crest cells with high plasticity. Prasmickaite et $\mathrm{al}^{34}$ believe that there is no significant difference in proliferation and clone formation ability between ALDH+ and ALDH- CM cells. Histopathological evidence supports the view that most $\mathrm{CM}$ actually occurs in the basal cell layer of the epidermis rather than in the dermis or hair follicles where CM-CSC is most concentrated. About $27 \%$ of unselected CM cells can form tumors in NOD/SCID mice, while breast cancer cells need more than or equal to 100 tumor cells selected by CSC markers to form tumor. ${ }^{55}$ Doherty also proved that the expression of $\mathrm{ALDH}$, CD44 and CD133 did not select CSC-like subsets in UM cell line or short-term cultured primary UM cells. There was a lack of cellular hierarchical structure in the cell line, and all cultured cells could promote tumor progression. $^{48}$ Both UM cell lines and short-term cultured UM cells were cultured in ocular melanoma medium (OC. Mel.) and tumor stem cell culture medium (CSCM) showed adherent monolayer of amelanotic cells and form spheres of melanotic cells, respectively. When melanotic cells that had grown in CSCM were reseeded into OC. Mel., they became adherent amelanotic cells, while the adherent amelanotic cells were reseeded into CSCM, UM cells detached and formed spheres, indicating that the plasticity of UM cells is higher. Maybe the highly malignant cells in UM were transformed from less malignant cells, but not directly related to $\mathrm{CSC}$. More and more studies have found that there is phenotypic plasticity in melanoma cells. MITF, as a key regulatory factor in this phenomenon, plays an important role in cell differentiation, cell cycle, DNA damage repair and senescence. ${ }^{56}$

UM mainly spread by hematogenous metastasis, nestin-positive circulating tumor cells were found in the peripheral blood of metastatic UM patients, ${ }^{57}$ and the expression of nestin in metastatic UM tissues was also higher than that of primary $\mathrm{UM}^{25}$ This seems to suggest that there is some connection between metastatic UM and CSC. Pan et al injected UM line cells into the NOG mice intrasplenically to verify the transfer capability of UM. The result showed that the drug could not only repress UM-CSC properties but also decrease the number and the size of metastatic nodules on the surface of the livers. ${ }^{12,52}$ However, there is no direct evidence to prove that metastatic UM is caused by CSC. Moreover, whether UM-CSC exists or not and how to identify it all need further experimental research. 


\section{Conclusion}

The concept of CSC provides a new direction for cancer treatment. As the source of tumorigenesis and development, targeted therapy of CSC may improve the effect of clinical diagnosis and treatment in the future. However, at present, the isolation, purification and differentiation pathway tracing of CSC are not yet mature. UM is a highly malignant tumor derived from the neural crest in the eye, its high heterogeneity brings a lot of difficulties to the research and treatment of CSC, but we believed that the progress of science and technology and the deepening of research in the field of CSC will bring us new ideas for the treatment of this disease.

\section{Acknowledgments}

The authors thank those who participated in the present study.

\section{Funding}

This study was supported by the National Natural Science Foundation of China (81570891); Beijing Natural Science Foundation (7151003); the Beijing Municipal Administration of Hospitals' Ascent Plan (DFL20150201); Advanced Health Care Professionals Development Project of Beijing Municipal Health Bureau (2014-2-003); The Capital Health Research and Development of Special (20161-2051); Beijing Municipal Administration of Hospitals Clinical Medicine Development of Special Funding Support (ZYLX201307); Science \& Technology Project of Beijing Municipal Science \& Technology Commission (Z181100001818003, Z151100001615052).

\section{Disclosure}

The authors report no conflicts of interest for this work.

\section{References}

1. Hu DN, Yu GP, McCormick SA, Schneider S, Finger PT. Populationbased incidence of uveal melanoma in various races and ethnic groups. Am J Ophthalmol. 2005;140(4):612.e1-612.e8. doi:10.1016/j.ajo.2005. 05.034

2. Houtzagers LE, Wierenga APA, Ruys AAM, Luyten GPM, Jager MJ. Iris colour and the risk of developing uveal melanoma. Int J Mol Sci. 2020;21(19):7172. doi:10.3390/ijms21197172

3. Ghazawi FM, Darwich R, Le M, et al. Uveal melanoma incidence trends in Canada: a national comprehensive population-based study. $\mathrm{Br}$ $J$ Ophthalmol. 2019;103(12):1872-1876. doi:10.1136/bjophthalmol2018-312966

4. Aronow ME, Topham AK, Singh AD. Uveal melanoma: 5-year update on incidence, treatment, and survival (SEER 1973-2013). Ocul Oncol Pathol. 2018;4(3):145-151. doi:10.1159/000480640
5. Kaštelan S, Antunica AG, Oresković LB, et al. Immunotherapy for uveal melanoma - current knowledge and perspectives. Curr Med Chem. 2019. doi:10.2174/0929867326666190704141444

6. Komatsubara KM, Carvajal RD. Immunotherapy for the treatment of uveal melanoma: current status and emerging therapies. Curr Oncol Rep. 2017;19(7):45. doi:10.1007/s11912-017-0606-5

7. Clarke MF, Dick JE, Dirks PB, et al. Cancer stem cells - Perspectives on current status and future directions: AACR workshop on cancer stem cells. Cancer Res. 2006;66(19):9339-9344. doi:10.1158/00085472.CAN-06-3126

8. Murphy GF, Wilson BJ, Girouard SD, Frank NY, Frank MH. Stem cells and targeted approaches to melanoma cure. Mol Aspects Med. 2014;39(1):33-49. doi:10.1016/j.mam.2013.10.003

9. Yu Y, Ramena G, Elble RC. The role of cancer stem cells in relapse of solid tumors. Front Biosci (Elite Ed). 2012;4(2):1528-1541. doi: $10.2741 / 478$

10. Prasetyanti PR, Medema JP. Intra-tumor heterogeneity from a cancer stem cell perspective. Mol Cancer. 2017;16(1). doi:10.1186/s12943017-0600-4

11. Jin B, Zhang P, Zou H, et al. Verification of EZH2 as a druggable target in metastatic uveal melanoma. Mol Cancer. 2020;19(1):1-15. doi:10.1186/s12943-020-01173-x

12. Zhou J, Liu S, Wang Y, et al. Salinomycin effectively eliminates cancer stem-like cells and obviates hepatic metastasis in uveal melanoma. Mol Cancer. 2019;18(1):159. doi:10.1186/s12943-0191068-1

13. Djirackor L, Kalirai H, Coupland SE, Petrovski G. CD166high uveal melanoma cells represent a subpopulation with enhanced migratory capacity. Investig Ophthalmol Vis Sci. 2019;60(7):2696-2704. doi:10.1167/iovs.18-26431

14. Kalirai H, Damato BE, Coupland SE. Uveal melanoma cell lines contain stem-like cells that self-renew, produce differentiated progeny, and survive chemotherapy. Investig Ophthalmol Vis Sci. 2011;52(11):8458-8466. doi:10.1167/iovs.11-7379

15. Onken MD, Worley LA, Ehlers JP, Harbour JW. Gene expression profiling in uveal melanoma reveals two molecular classes and predicts metastatic death. Cancer Res. 2004;64(20):7205-7209. doi:10.1158/0008-5472.CAN-04-1750

16. Chang SH, Worley LA, Onken MD, Harbour JW. Prognostic biomarkers in uveal melanoma: evidence for a stem cell-like phenotype associated with metastasis. Melanoma Res. 2008;18(3):191-200. doi:10.1097/CMR.0b013e3283005270

17. Matatall KA, Agapova OA, Onken MD, et al. BAP1 deficiency causes loss of melanocytic cell identity in uveal melanoma. $B M C$ Cancer. 2013;13(1). doi:10.1186/1471-2407-13-371.

18. Kuznetsov JN, Aguero TH, Owens DA, et al. BAP1 regulates epigenetic switch from pluripotency to differentiation in developmental lineages giving rise to BAP1-mutant cancers. Sci Adv. 2019;5(9): eaax1738. doi:10.1126/sciadv.aax 1738

19. Herold-Mende C, Mock A. Microenvironment and brain tumor stem cell maintenance: impact of the niche. Anticancer Agents Med Chem. 2014;14(8):1065-1074. doi:10.2174/1871520614666 140825103636

20. Leon G, MacDonagh L, Finn SP, Cuffe S, Barr MP. Cancer stem cells in drug resistant lung cancer: targeting cell surface markers and signaling pathways. Pharmacol Ther. 2016;158:71-90. doi:10.1016/ j.pharmthera.2015.12.001

21. Peiris-Pagès $M$, Martinez-Outschoorn UE, Pestell RG, Sotgia F, Lisanti MP. Cancer stem cell metabolism. Breast Cancer Res. 2016;18(1):55. doi:10.1186/s13058-016-0712-6

22. Tirosh I, Venteicher AS, Hebert C, et al. Single-cell RNA-seq supports a developmental hierarchy in human oligodendroglioma. Nature. 2016;539(7628):309-313. doi:10.1038/nature20123

23. Thill M, Berna MJ, Grierson R, et al. Expression of CD133 and other putative stem cell markers in uveal melanoma. Melanoma Res. 2011;21(5):405-416. doi:10.1097/CMR.0b013e328348db10 
24. Grossniklaus HE, Zhang Q, You S, et al. Metastatic ocular melanoma to the liver exhibits infiltrative and nodular growth patterns. Hum Pathol. 2016;57:165-175. doi:10.1016/j.humpath.2016.07.012

25. Djirackor L, Shakir D, Kalirai H, Petrovski G, Coupland SE. Nestin expression in primary and metastatic uveal melanoma - possible biomarker for high-risk uveal melanoma. Acta Ophthalmol. 2018;96 (5):503-509. doi:10.1111/aos.13645

26. Valyi-Nagy K, Kormos B, Ali M, Shukla D, Valyi-Nagy T. Stem cell marker CD271 is expressed by vasculogenic mimicryforming uveal melanoma cells in three-dimensional cultures. Mol Vis. 2012;18: (March):588-592.

27. Bergeron MA, Champagne S, Gaudreault M, Deschambeault A, Landreville S. Repression of genes involved in melanocyte differentiation in uveal melanoma. Mol Vis. 2012;18:1813-1822.

28. Radberger P, Radberger A, Vladimir JNB, Seregard S, Economou MA. JARID1B protein expression and prognostic implications in uveal melanoma. Investig Ophthalmol Vis Sci. 2012;53 (8):4442-4449. doi:10.1167/iovs.11-9296

29. Roudi R, Korourian A, Shariftabrizi A, Madjd Z. Differential expression of cancer stem cell markers ALDH1 and CD133 in various lung cancer subtypes. Cancer Invest. 2015;33(7):294-302. doi:10.3109/ 07357907.2015 .1034869

30. Jang JW, Song Y, Kim SH, Kim J, Seo HR. Potential mechanisms of CD133 in cancer stem cells. Life Sci. 2017;184:25-29. doi:10.1016/j. Ifs. 2017.07 .008

31. Madjd Z, Erfani E, Gheytanchi E, et al. Expression of CD133 cancer stem cell marker in melanoma: a systematic review and meta-analysis. Int $J$ Biol Markers. 2016;31(2):e118e125. doi:10.5301/jbm.5000209

32. Lunter PC, van Kilsdonk JWJ, van Beek H, et al. Activated leukocyte cell adhesion molecule (ALCAM/CD166/MEMD), a novel actor in invasive growth, controls matrix metalloproteinase activity. Cancer Res. 2005;65(19):8801-8808. doi:10.1158/0008-5472.CAN-05-0378

33. Boiko AD, Razorenova OV, Van De Rijn M, et al. Human melanoma-initiating cells express neural crest nerve growth factor receptor CD271. Nature. 2010;466(7302):133-137. doi:10.1038/ nature09161

34. Prasmickaite L, Engesæter B, Skrbo N, et al. Aldehyde dehydrogenase (ALDH) activity does not select for cells with enhanced aggressive properties in malignant melanoma. PLoS One. 2010;5(5): e10731. doi:10.1371/journal.pone.0010731

35. Sarvi S, Crispin R, Lu Y, et al. ALDH1 bio-activates nifuroxazide to eradicate ALDH high melanoma-initiating cells. Cell Chem Biol. 2018;25(12):1456-1469.e6. doi:10.1016/j.chembiol.2018.09.005

36. Yue L, Huang ZM, Fong S, et al. Targeting ALDH1 to decrease tumorigenicity, growth and metastasis of human melanoma. Melanoma Res. 2015;25(2):138-148. doi:10.1097/CMR.0000000 000000144

37. Santini R, Pietrobono S, Pandolfi S, et al. SOX2 regulates self-renewal and tumorigenicity of human melanoma-initiating cells. Oncogene. 2014;33(38):4697-4708. doi:10.1038/onc.2014.71

38. Schaefer SM, Segalada C, Cheng PF, et al. Sox2 is dispensable for primary melanoma and metastasis formation. Oncogene. 2017;36 (31):4516-4524. doi:10.1038/onc.2017.55

39. Cesarini V, Guida E, Todaro F, et al. Sox2 is not required for melanomagenesis, melanoma growth and melanoma metastasis in vivo. Oncogene. 2017;36(31):4508-4515. doi:10.1038/onc.2017.53

40. Wang S, Tang L, Lin J, et al. ABCB5 promotes melanoma metastasis through enhancing $\mathrm{NF}-\kappa \mathrm{B}$ p65 protein stability. Biochem Biophys Res Commun. 2017;492(1):18-26. doi:10.1016/j. bbrc.2017.08.052
41. Louphrasitthiphol P, Chauhan J, Goding CR. ABCB5 is activated by MITF and $\beta$-catenin and is associated with melanoma differentiation. Pigment Cell Melanoma Res. 2020;33(1):112-118. doi:10.1111/ pcmr. 12830

42. Monzani E, Facchetti F, Galmozzi E, et al. Melanoma contains CD133 and ABCG2 positive cells with enhanced tumourigenic potential. Eur J Cancer. 2007;43(5):935-946. doi:10.1016/j.ejca.20 07.01 .017

43. Gunawan M, Venkatesan N, Loh JT, et al. The methyltransferase Ezh2 controls cell adhesion and migration through direct methylation of the extranuclear regulatory protein talin. Nat Immunol. 2015;16 (5):505-516. doi:10.1038/ni.3125

44. Fisher ML, Adhikary G, Grun D, Kaetzel DM, Eckert RL. The Ezh2 polycomb group protein drives an aggressive phenotype in melanoma cancer stem cells and is a target of diet derived sulforaphane. Mol Carcinog. 2016;55(12):2024-2036. doi:10.1002/mc.22448

45. Roesch A, Fukunaga-Kalabis M, Schmidt EC, et al. A temporarily distinct subpopulation of slow-cycling melanoma cells is required for continuous tumor growth. Cell. 2010;141(4):583-594. doi:10.1016/j. cell.2010.04.020

46. Kumar SM, Liu S, Lu H, et al. Acquired cancer stem cell phenotypes through Oct4-mediated dedifferentiation. Oncogene. 2012;31 (47):4898-4911. doi:10.1038/onc.2011.656

47. Wickremesekera AC, Brasch HD, Lee VM, et al. Expression of cancer stem cell markers in metastatic melanoma to the brain. J Clin Neurosci. 2019;60:112-116. doi:10.1016/j.jocn.2018.10.068

48. Doherty RE, Sisley K, Hammond DW, Rennie IG, Cross NA. Phenotypic plasticity in uveal melanoma is not restricted to a tumor subpopulation and is unrelated to cancer stem cell characteristics. Investig Ophthalmol Vis Sci. 2017;58(12):5387-5395. doi:10.1167/ iovs.17-22272

49. Jin Y, Zhang P, Wang Y, et al. Neddylation blockade diminishes hepatic metastasis by dampening cancer stem-like cells and angiogenesis in uveal melanoma. Clin Cancer Res. 2018;24 (15):3741-3754. doi:10.1158/1078-0432.CCR-17-1703

50. Wang Y, Liu M, Jin Y, Jiang S, Pan J. In vitro and in vivo anti-uveal melanoma activity of JSL-1, a novel HDAC inhibitor. Cancer Lett. 2017;400(April):47-60. doi:10.1016/j.canlet.2017.04.028

51. Zhou J, Jin B, Jin Y, Liu Y, Pan J. The antihelminthic drug niclosamide effectively inhibits the malignant phenotypes of uveal melanoma in vitro and in vivo. Theranostics. 2017;7(6):1447-1462. doi:10.7150/thno.17451

52. Zhang J, Liu S, Ye Q, Pan J. Transcriptional inhibition by CDK7/9 inhibitor SNS-032 abrogates oncogene addiction and reduces liver metastasis in uveal melanoma. Mol Cancer. 2019;18(1):140. doi:10.1186/s12943-019-1070-7

53. Rundle P. Treatment of posterior uveal melanoma with multi-dose photodynamic therapy. $\mathrm{Br} J$ Ophthalmol. 2014;98(4):494-497. doi:10.1136/bjophthalmol-2013-304432

54. Ma YW, Liu YZ, Pan JX. Verteporfin induces apoptosis and eliminates cancer stem-like cells in uveal melanoma in the absence of light activation. Am J Cancer Res. 2016.

55. Quintana E, Shackleton M, Sabel MS, et al. Efficient tumour formation by single human melanoma cells. Nature. 2008;456 (7222):593-598. doi:10.1038/nature07567

56. Rambow F, Marine JC, Goding CR. Melanoma plasticity and phenotypic diversity: therapeutic barriers and opportunities. Genes Dev. 2019;33(19-20):1295-1318. doi:10.1101/gad.329771.119

57. Fusi A, Reichelt U, Busse A, et al. Expression of the stem cell markers nestin and CD133 on circulating melanoma cells. J Invest Dermatol. 2011;131(2):487-494. doi:10.1038/jid.2010.285 


\section{Publish your work in this journal}

OncoTargets and Therapy is an international, peer-reviewed, open access journal focusing on the pathological basis of all cancers, potential targets for therapy and treatment protocols employed to improve the management of cancer patients. The journal also focuses on the impact of management programs and new therapeutic agents and protocols on patient perspectives such as quality of life, adherence and satisfaction. The manuscript management system is completely online and includes a very quick and fair peer-review system, which is all easy to use. Visit http://www.dovepress.com/ testimonials.php to read real quotes from published authors. 